\title{
¿Por qué los estudiantes de colegios públicos y privados de Costa Rica obtienen distintos resultados académicos?
}

\author{
Gregorio Giménez,* Geovanny Castro Aristizábal**
}

Perfiles Latinoamericanos

Flacso México

\begin{abstract}
Resumen
En este artículo se busca identificar qué causa las diferencias en resultados académicos entre colegios costarricenses públicos y privados empleando la técnica de descomposición de Oaxaca-Blinder, aplicada a la base PISA 2012. La conclusión es que las diferencias, por orden de importancia, se deben a: a) las características de los hogares, b) los recursos de los centros, c) las características de los estudiantes, $\mathrm{y} d$ ) el ambiente de trabajo en los centros. Pero también porque hay diferencias en características y factores que se aprovechan de modo distinto. Así, si los estudiantes de escuelas públicas poseen en conjunto peores características, saben sacarles mejor partido, aunque los estudiantes de los colegios privados sabrían aprovechar mejor el ambiente de trabajo en este tipo de centros educativos.
\end{abstract}

\begin{abstract}
In this paper we use the Oaxaca-Blinder decomposition methodology, applied to the PISA 2012 database, in order to identify the causes of the differences in academic results between public and private Costa Rican schools. In order of importance, these are caused by differences in: a) family characteristics, $b$ ) school resources, $c$ ) student characteristics, and d) working environment in the schools. The differences in the results are not only explained by the differences in characteristics and factors, but also by the differences in the way they are used. So, while students in public schools have collectively worst characteristics, they make better use of them. However, students in private schools obtain more output from the working environment in their schools.
\end{abstract}

Palabras clave: resultados escolares, educación pública, educación privada, Costa Rica, PISA, descomposición Oaxaca-Blinder, funciones de producción educativa.

Keywords: Academic achievement, public education, private education, Costa Rica, PISA, OaxacaBlinder decomposition, educational production functions.

* Doctor en Economía por la Universidad de Zaragoza. Profesor de Economía, Universidad de Zaragoza.

** Maestro en Ciencias Sociales por la Pontificia Universidad Javeriana (Cali, Colombia). 


\section{Introducción ${ }^{1}$}

e acuerdo a la información de la Megabase de datos georreferenciados de centros educativos de Costa Rica (2000-2012), el número de alumnos matriculados en 2012 en educación secundaria fue de 359138 estudiantes. De los que 317950 correspondieron a centros públicos, 28589 a centros privados y 12599 a centros subvencionados. A pesar del reducido peso que la educación privada tiene en el país, este tipo de instituciones cuenta con buena reputación, lo que se basa en la calidad de sus recursos educativos y profesorado. Este hecho origina un intenso debate acerca de si los alumnos que asisten a centros privados reciben mejor educación. Una educación de más calidad explicaría por qué dichos estudiantes sufren en menor medida el fracaso escolar y obtienen mejores resultados en pruebas estandarizadas.

No obstante, es importante señalar que las diferencias en puntuación en este tipo de pruebas no implican necesariamente que la calidad de la educación de los colegios públicos sea peor, o que dichos centros dispongan de menos recursos o tengan peores profesores. Hay que tener en cuenta que las características socioeconómicas de los estudiantes de los centros públicos y privados son distintas, e influyen en la elección de los colegios (sesgos de selección). Así, es más probable que, en promedio, los alumnos de centros privados provengan de familias con más recursos económicos y mayores niveles educativos. De este modo, el nivel socioeconómico de la familia, las facilidades educativas existentes en el hogar, o los efectos externos asociados a tener por compañeros estudiantes que pertenecen a estos entornos, serían las causas que explicarían los mejores resultados de los alumnos de colegios privados.

El presente trabajo pretende estimar, en primer lugar, cuáles son las diferencias en rendimiento entre centros públicos y privados costarricenses, una vez que se ha controlado un amplio conjunto de determinantes. Además, se pretende ir un paso más allá de la mayoría de trabajos en economía de la educación y, a través del uso de la técnica de descomposición de Oaxaca-Blinder, analizar las causas que subyacen a estas diferencias. Dada la escasez de este tipo de investigaciones en economías en desarrollo en general, y en América Latina en particular, este artículo supone una contribución importante. En este sentido, la investigación se beneficia de las posibilidades metodológicas que ofrece una base tan rica como la elaborada por el Program for International Student Assessment (PISA), en su edición de 2012.

1 Los autores desean agradecer a Estado de la Educación (Costa Rica) el inestimable apoyo financiero y técnico prestado para el desarrollo de la investigación. 
El análisis llevado a cabo revela que las diferencias obedecen a las distintas dotaciones en los inputs incluidos en la función de producción educativa: características del estudiante, características del hogar, diferencias en recursos y ambiente de trabajo en los centros. Asimismo, también se constatan diferencias en el aprovechamiento de dichos factores que contribuyen a ahondar las que se dan en rendimiento.

El trabajo queda estructurado del siguiente modo. A esta introducción le sigue una segunda sección, donde se contextualiza la investigación dentro de la literatura sobre economía de la educación. En la tercera sección, i) se expone el modelo base que se utilizará en las estimaciones, ii) se explica el método de descomposición de Oaxaca-Blinder usado en la investigación empírica, y iii) se realiza una serie de consideraciones metodológicas sobre las técnicas de estimación empleadas. En una cuarta sección se ofrecen los resultados del análisis econométrico, en el que se constatan las diferencias en desempeño escolar entre centros públicos y privados y se descomponen en función de i) las características de los alumnos, hogares y centros, ii) el aprovechamiento de estas características, y iii) la interacción del efecto características y el efecto aprovechamiento. En un apartado final, se presentan las conclusiones del trabajo y sus implicaciones en términos de equidad y diseño de la política educativa.

\section{Contextualización: educación pública, educación privada y resultados académicos}

¿Asistir a un centro privado condiciona los resultados escolares? La evidencia empírica en economía de la educación es ambigua. Los trabajos teóricos suelen tener en cuenta dos aspectos al estudiar los efectos de la educación privada sobre el desempeño escolar.

En primer lugar, los centros privados pueden tener mayor grado de autonomía en la gestión, lo que les permitiría ser más eficientes. Además, si disponen de mayor presupuesto, podrán contratar a profesores más cualificados, tener menos alumnos por clase, brindar más actividades y ofrecer mejor infraestructura —en términos de aulas, laboratorios, equipamientos o materiales- Sin embargo, no existe una evidencia empírica concluyente sobre si una mayor autonomía de los centros escolares mejora el rendimiento académico. Así, Steinberg (2014), que utiliza datos del Autonomous Management and Performance Schools Program para escuelas públicas de Chicago, encuentra una relación directa y estadísticamente significativa entre obtención de mayor autonomía y mejora del rendimiento escolar en lectura, pero no la encuentra en el caso de matemáticas; e incluso advierte que, cuando los colegios reciben nuevas competencias, los efectos de la 
autonomía pueden tardar tiempo en verse acompañados de mejoras en resultados. Otros autores también apuntan la ambigüedad de la relación al utilizar muestras de datos internacionales. Hanushek et al. (2013) encuentran resultados mixtos en una amplia muestra de 42 países que utiliza datos del PISA (2000-2009). De acuerdo a estos autores, la mayor autonomía afectaría negativamente a los países en desarrollo y con peores resultados, pero sería positiva para los países con mayores niveles de desarrollo y mejores resultados. Por otra parte, Benton (2014), que trabaja con datos del PISA (2009), no concluye que la relación entre mayor autonomía y mejora en resultados sea significativa, una vez que se examinan por separado los datos de escuelas públicas y privadas.

En segundo lugar, la existencia de centros privados produce un incremento de la competencia entre centros educativos, incluidos los públicos, la cual puede influir positivamente sobre los resultados académicos de los alumnos de ambos tipos de centro (Woessmann et al., 2009; West \& Woessman, 2010). No obstante, OCDE (2014) evidencia que la mayor competencia entre centros no se relaciona con mejores resultados en las pruebas de matemáticas según el PISA (2012).

En cuanto a la literatura que trata de estimar directamente los efectos de la titularidad del centro sobre los resultados escolares, hay que señalar que tampoco ofrece resultados concluyentes (Bishop \& Woessmann, 2004). Así, diversos trabajos advierten que el supuesto efecto positivo de la educación privada se desvanece una vez que se introducen las características socioeconómicas de los alumnos como variables de control. Dentro de esta línea de estudios, Calero \& Escardíbul (2007) apuntan que, en el caso de España, las brechas en rendimiento escolar a favor de los centros privados obedecerían más a características asociadas al estudiante y a su familia. Del mismo modo, para el caso de Estados Unidos, Gamoran (1996) y Altonji et al. (2005) encuentran que, una vez que se controla por un elevado número de características observables e inobservables de los alumnos, el nexo entre asistencia a centros privados y resultados académicos se vuelve débil. Respecto a estudios que tienen en cuenta una muestra amplia de países a partir de datos del PISA, Dronkers \& Robert (2005) hallan nueva evidencia de que existen sesgos de selección en una muestra de 19 países de la OCDE. De este modo, serían las variables de índole social las que estarían condicionando los mejores resultados en matemáticas de los alumnos que acuden a colegios privados. Una vez que se controla por las variables socioeconómicas, las escuelas privadas obtendrían peores resultados que las públicas. Si bien, excepcionalmente, las escuelas privadas dependientes del gobierno alcanzarían mejores resultados. En la misma línea irían las conclusiones que ofrece OCDE (2011), las cuales encuentran que 1) los estudiantes de escuelas públicas obtienen los mismo resultados que los de las privadas, una vez que se corrige por el factor socioeconómico, y 2) que los países con una mayor propor- 
ción de colegios privados no obtienen mejores resultados en las pruebas PISA. Algunos trabajos — como los de Newhouse \& Beegle (2006) para Indonesia, y Kirjavainen \& Loikkanen (1998) para Finlandia - incluso encuentran que el efecto de la educación privada sobre los resultados académicos es negativo.

Con todo, la mayor parte de los análisis revelan un vínculo positivo entre educación privada y un amplio conjunto de factores relacionados con el éxito escolar, como son los resultados en pruebas de conocimiento, la finalización de los ciclos escolares, la realización de estudios superiores o los mayores salarios cobrados en el mercado laboral. Así, por ejemplo, Coleman et al. (1982) y Coleman \& Hoffer (1987) —el primero para una muestra de corte transversal, y el segundo utilizando una comparación temporal de los resultados de estudiantes en décimo y duodécimo grado - constatan el efecto positivo de la asistencia a centros privados sobre el rendimiento académico para el caso de Estados Unidos.

Muchos trabajos coinciden en el carácter religioso que en muchas ocasiones tienen los centros privados, y cómo esto puede influir en su gestión y métodos docentes. ${ }^{2}$ Schultz \& McDonald (2013) encuentran que, una vez que se ha corregido por otras variables explicativas, los estudiantes de colegios privados estadounidenses gestionados por órdenes católicas obtienen mejores resultados que los de los públicos. También para Estados Unidos, Figlio \& Stone (2001) constatan que los colegios privados religiosos mejoran los resultados de los alumnos que pertenecen a minorías étnicas. Ello estaría en línea con lo hallado por Neal (1997), quien concluye que la asistencia a instituciones católicas beneficia sobre todo a las minorías étnicas urbanas, ya que las escuelas públicas a las que estos estudiantes pueden acceder son de calidad baja. Estos efectos se repiten en estudiantes de raza blanca y entornos urbanos, aunque serían menos intensos. El efecto positivo de los colegios religiosos de igual modo se valida si se aplican técnicas econométricas más sofisticadas, que permiten el uso de variables instrumentales y métodos de estimación en dos etapas. De esta forma, se tiene en cuenta que la variable asistir a un colegio religioso puede estar relacionada con otras no recogidas en el modelo, pero que pueden afectar a los resultados escolares (Cohen-Zada \& Sander, 2007).

En cuanto a los resultados empíricos fuera del ámbito escolar estadounidense, existen muchos ejemplos relevantes, por ejemplo, los trabajos de Green et al. (2012) y Dearden et al. (2010), los cuales señalan que los alumnos británicos que asisten a centros privados tienen, en promedio, más probabilidades de alcanzar niveles educativos superiores, y obtener mejores resultados académicos y mayores salarios. Para Holanda, Levin (2002) evidencia los mejores resultados de los alumnos de colegios católicos privados. Vella (1999), por su parte,

2 Véase la síntesis que realizan Sander \& Cohen-Zada (2010).

G. Giménez, G. Castro Aristizábal | ¿Por qué los estudiantes de colegios públicos y privados de Costa Rica obtienen distintos resultados académicos? | Perfiles Latinoamericanos | FLACso México 
demuestra que, en Australia, los alumnos que han estudiado en dicho tipo de instituciones presentan más probabilidades de terminar el bachillerato, asistir a la universidad o encontrar empleo. Y respecto a trabajos que utilizan una muestra amplia de países, West \& Woessmann (2010) estiman que, a medida que aumenta la importancia del sector educativo privado, los países mejoran los resultados escolares generales. Mientras que Fuchs \& Woessmann (2007) constatan este efecto positivo utilizando resultados del PISA.

Los efectos positivos de asistir a centros privados se han constatado además en países en desarrollo. Jimenez \& Cox (1991) lo hacen para estudiantes de Tanzania y Colombia y Jiménez et al. (1991) para los de Colombia, República Dominicana, Filipinas, Tanzania y Tailandia. Ambos trabajos controlan por factores socieconómicos y encuentran una relación positiva entre la asistencia a instituciones privadas y las notas conseguidas en los tests de matemáticas y lectura. Lassibille \& Tan (2001), sin embargo, no hallan ese efecto positivo de la educación privada en el caso de Tanzania, una vez que se corrigen los sesgos de selección.

En cuanto a los trabajos enfocados en países latinoamericanos, Lockheed \& Bruns (1990) constatan, a través de un estudio empírico financiado en Brasil por el Banco Mundial y con datos de 2611 alumnos y 62 escuelas, que - una vez descontados los efectos del tamańo familiar, la edad, el sexo y el número de horas de estudio- los alumnos de colegios privados obtienen mejores resultados en las pruebas de matemáticas, pero no en lengua portuguesa. También para Brasil, Oliveira et al. (2013) no encuentran evidencia del efecto positivo de los colegios privados. Utilizando regresiones cuantílicas y contrafactuales concluyen que los alumnos de octavo grado con malos resultados académicos en colegios públicos los obtendrían incluso peores si acudieran a centros privados. De igual forma, dichos estudios resaltan la importancia del entorno familiar en estos resultados. Existe evidencia de que el entorno socieconómico familiar es tan importante como el de la escuela. Así, Formichella (2011) aplica técnicas multinivel a datos PISA (2006) de estudiantes argentinos y concluye que la relación entre la estructura administrativa de la escuela y el rendimiento escolar de los estudiantes desaparece cuando se toma en consideración el entorno socioeconómico del centro.

Respecto a Costa Rica, las investigaciones que utilizan técnicas econométricas para evaluar los factores que condicionan los resultados educativos son ya de por sí muy escasos. Los recientes trabajos de Montero et al. (2012) y Fernández \& Del Valle (2013), ambos con datos del PISA (2009), constituyen una excepción. El primero muestra que, después de haber controlado por una amplia gama de características de los alumnos y centros, los estudiantes que asisten a centros públicos diurnos obtienen peores resultados que los alumnos de centros privados, y estas diferencias son significativas en un nivel del 10\%; 
aunque, debe indicarse, las diferencias entre centros no son significativas para colegios públicos técnicos, nocturnos y colegios rurales o de telesecundaria. Por su parte, Fernández \& Del Valle (2013) determinan que existen diferencias relevantes entre las puntuaciones de los alumnos de colegios públicos y privados, a favor de los colegios privados, y estas son significativas al 5\% en las pruebas de matemáticas y lectura, pero no en ciencias. Por tanto, la ambigüedad en los resultados señalada en la literatura empírica se da también para Costa Rica.

La escasez de estudios empíricos para América Latina en general y para Costa Rica en particular, así como la necesidad de nuevos enfoques que aporten solidez metodológica, dada la ambigüedad de los resultados de los trabajos internacionales, serían las causas que nos llevan a desarrollar el análisis que planteamos a continuación.

\section{Modelización y consideraciones metodológicas}

\section{La función de producción educativa usada en las estimaciones}

Para cuantificar el efecto de la educación privada en los resultados escolares, diseñamos una función de producción educativa que servirá de modelo base para las estimaciones. Estas funciones permiten analizar la eficiencia en la asignación y uso de los recursos educativos al establecer empíricamente, mediante técnicas de regresión, la relación entre factores o inputs educativos y los resultados u output. Estos últimos pueden medirse como tasas de matriculación, ańos de estudio, abandono escolar o, más habitualmente, conocimientos, evaluados con pruebas estandarizadas. Su uso, similar al de una función de producción empresarial, pero aplicado al ámbito de la educación, facilita la adopción de decisiones sobre qué recursos deben ser utilizados y en qué cuantía, con el fin de optimizar el proceso de aprendizaje de los alumnos.

Aunque la utilización de funciones de producción educativa es habitual en la economía de la educación, existe todavía una carencia notable de trabajos empíricos en América Latina, entre otros motivos, por las dificultades metodológicas y la escasez de datos. La falta de datos se ha paliado en los últimos años gracias a proyectos como el PISA, el cual brinda una de las bases internacionales de datos educativos más completas, con información sobre resultados en pruebas de matemáticas, comprensión lectora y ciencias para una amplia muestra de alumnos de quince años. Incluye también gran cantidad de datos sobre sus características, las de su entorno familiar y las de los centros en los que estudian. En su edición de 2012, participaron ocho naciones latinoamericanas: Argentina, Brasil, Chile, Colombia, Costa Rica, México, Perú y Uruguay. 
La función de producción educativa que va a servir de base a nuestras estimaciones se formula específicamente como:

$$
\begin{aligned}
& R_{i}=\beta_{0}+\beta_{1} P_{i}+\beta_{2} X_{i}+\varepsilon_{i} \\
& \text { Con: } \operatorname{COV}\left(P_{i}, \varepsilon_{i}\right)=0 \\
& \operatorname{COV}\left(X_{i}, \varepsilon_{i}\right)=0
\end{aligned}
$$

Donde $R_{i}$ representa los resultados del alumno i-ésimo en las pruebas de matemáticas, lectura y ciencias. $P_{i}$ es una variable dicotómica que toma valores $(\mathrm{P}=1)$ si la escuela es privada, o $(\mathrm{P}=0)$ si es pública. $X_{i}$ es un vector que incluye las variables de control que condicionan los resultados escolares. Así, $\beta_{1}$ indica la diferencia en resultados que se debe a que la institución educativa sea pública o privada, una vez que se ha controlado por el resto de variables. Por último, $\varepsilon_{i}$ es un término de error propio de cada alumno. Puede tener origen en la omisión de variables importantes, errores de medición o en una errónea especificación funcional.

El éxito en la cuantificación y explicación de las diferencias en desempeño escolar entre centros públicos y privados dependerá de la precisión en el planteamiento de la función de producción educativa diseñada. Identificar las variables que permiten medir de manera precisa los inputs educativos es una tarea difícil. Las variables de control se suelen agrupar en tres grandes categorías (Harris, 2010): 1) características del estudiante, 2) características del hogar y 3) características del centro educativo. La elección de variables que incluiremos en cada categoría es habitual en la literatura empírica sobre economía de la educación, lo cual se ha recogido en Burtless (1996), Krueger (1999), Hanushek (2005), y Hanushek \& Welch (2006). El esquema 1 desagrega los elementos de cada categoría, así como las variables que los recogen en el análisis (detrás de corchete y en cursiva). Al final del artículo, un apéndice ofrece su descripción, fuentes y estadísticos principales.

De las características del estudiante se incluye el género y si cursó preescolar, además de ottras no observables, que resulta complicado captar con las variables que incluyen las bases de datos escolares. Entre estas últimas estarían la confianza o la habilidad innata de cada estudiante. Como se indica en el esquema, ambos componentes no observables se refuerzan. Para medir la primera, haremos uso de la respuesta a la pregunta "El estudiante piensa que no es bueno en matemáticas". ${ }^{3}$ Para la segunda, se utilizará como proxy la variable repetidor.

3 La utilización de la asignatura de matemáticas como base de la confianza del alumno se debe a que los informes del PISA enfatizan en cada edición en una materia, y PISA (2012) tuvo como foco las matemáticas. Por tanto, esa edición incluye un conjunto de cuestiones orientadas hacia esa asignatura, y no se recogen para otras. Además, las matemáticas constituyen una materia troncal e instrumental, y es razo- 
Entre las características del hogar se encuentra, en primer lugar, la educación de los progenitores, recogida a través de los años de estudio de la madre y del padre. En segundo lugar, los recursos del hogar, medidos con dos variables: un indice de riqueza en el hogar y un indice de posesiones culturales en el hogar.

Por último, las características del centro educativo comprenden un primer grupo de factores que engloban los recursos de infraestructuras y profesorado, recogidos por un indice de calidad de los recursos educativos del centro, la ratio alumnos/profesor y un indice de falta de profesores en el centro. Un segundo grupo recoge elementos institucionales. Aquí situamos la autonomía del centro educativo, que evaluamos mediante su condición de público o privado. Será la variable clave en la investigación empírica. Finalmente, dentro de los elementos institucionales se incluye el ambiente de trabajo en el centro educativo, que se aproxima a través de tres variables: un indice de relaciones profesor-alumno, la respuesta a la cuestión "El director del centro responde a si los profesores del centro muestran una moral alta" que indica la motivación del profesorado y un indice de disciplina en el centro.

Esquema 1. Inputs incluidos en la función de producción educativa

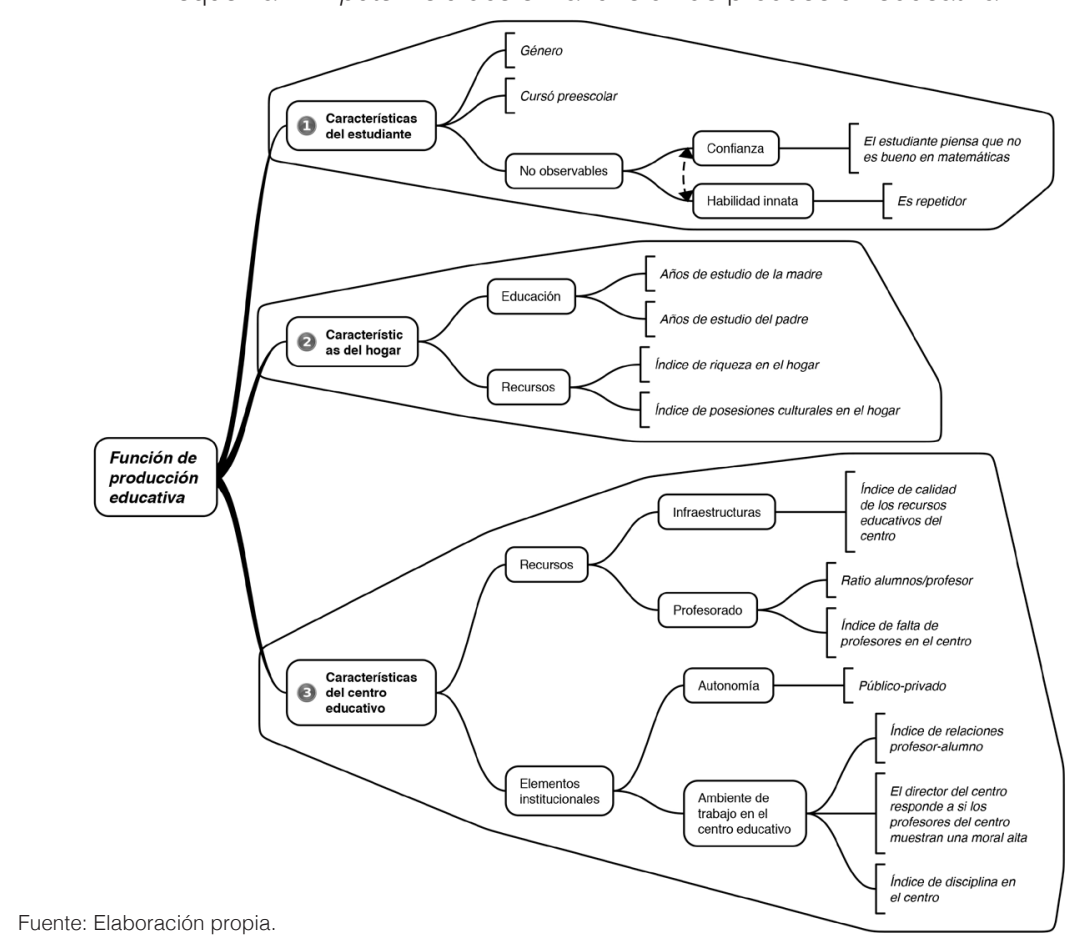

nable pensar que la confianza que el alumno posee en esta asignatura es una buena aproximación a su nivel de confianza general para el estudio de otras materias.

G. Giménez, G. Castro Aristizábal | ¿Por qué los estudiantes de colegios públicos y privados de Costa Rica obtienen distintos resultados académicos? | Perfiles Latinoamericanos | FLACso México 
La metodología fue desarrolla inicialmente en los artículos de Oaxaca (1973) y Blinder (1973). Desde entonces, la técnica ha sido utilizada en numerosas ocasiones, fundamentalmente en economía laboral. En los últimos años, su uso se ha extendido a otras áreas, como la economía de la educación. En dicho ámbito, y en América Latina, distintos trabajos la han empleado para analizar las causas de las diferencias en resultados escolares. Aunque en ellos la perspectiva metodológica es distinta a la de este artículo, ya que dichas investigaciones aplican la descomposición para el análisis de diferencias espaciales y temporales, y aquí la aplicamos en función de la titularidad del centro.

Así en el ámbito espacial, Ramos et al. (2012) utilizan las bases PISA 2006 y 2009 para analizar a qué se deben las diferencias en resultados entre colegios colombianos urbanos y rurales. El mayor rendimiento académico de las escuelas urbanas respecto de las rurales se explica más por las diferencias en sus características familiares que por los factores de escuela. También para el caso de Colombia, y con datos de las pruebas SABER 11 del Instituto Colombiano para el Fomento de la Educación Superior, Sánchez (2011) identifica las causas que están detrás de las brechas en desempeño escolar entre departamentos, en función de la composición étnica de sus estudiantes. Los estudiantes indígenas y afrocolombianos obtienen un rendimiento menor en un $9.6 \%$ en matemáticas y en un $4.7 \%$ en lenguaje, lo que se explica por sus grandes desventajas en dotaciones. Bellei et al. (2009) y Valenzuela et al. (2009) utilizan datos PISA 2006 e identifican los principales factores que diferencian el rendimiento escolar (matemáticas y lectura) de los estudiantes chilenos en relación a los uruguayos, españoles y polacos. Las brechas de Chile se explican por las diferencias en eficiencia educativa, es decir, por la menor productividad de sus características y recursos. Finalmente, Oreiro \& Valenzuela (2012) utilizan datos PISA con una perspectiva temporal para identificar las causas del pequeñísimo incremento en puntuación en matemáticas producido en las escuelas uruguayas entre 2003 y 2006. Concluyen que este se debe, fundamentalmente, a una mejora generalizada en la eficiencia en el uso de los recursos derivados de la recuperación económica en el periodo de estudio.

En el presente artículo, la descomposición ов brindará una potente herramienta de análisis para identificar cuáles serían las causas de las diferencias en desempeño académico entre centros públicos y privados costarricenses. En síntesis, la descomposición permite analizar a qué se deben las diferencias en resultados alcanzados por dos grupos de individuos - en nuestro caso, alumnos que asisten a colegios públicos y privados- por medio de dos componentes: una componente explicada por el modelo y una componente no expli- 
cada. ${ }^{4}$ Para ello, a partir de la función educativa propuesta, se calculan sendas regresiones para cada grupo y se analiza qué proporción de la diferencia en resultados alcanzados se debe a la existencia de características observables diferentes entre ambos. La componente explicada se calcula en función de las diferentes características propias de los individuos de cada grupo. En cuanto a la componente no explicada, es una componente residual asociada al aprovechamiento de las características grupales, y estaría recogiendo todo lo no explicado por el modelo planteado en función de aquellas. Además, incluiría la omisión de características relevantes no incorporadas o de componentes inobservables.

Las diferencias de los resultados promedio de dos grupos se pueden descomponer en tres factores. El primero corresponde con la que hemos denominado componente explicada y el segundo y tercero forman la componente no explicada. Así, la diferencia (D) en el valor esperado en los resultados entre los estudiantes de colegios privados (PRI) y públicos (PUB) quedaría como:

$$
\begin{gathered}
D=E\left(\text { Resultados }_{P R I}\right)-E\left(\text { Resultados }_{P U B}\right)= \\
\underbrace{\{\underbrace{\left.E\left(\mathrm{X}_{\mathrm{PRI}}\right)-\mathrm{E}\left(\mathrm{X}_{\mathrm{PUB}}\right)\right\}^{\prime} \beta_{\mathrm{PUB}}}_{\text {Efecto dotaciones }}}_{\text {Componente observada }}+
\end{gathered}
$$

$$
\underbrace{\underbrace{\left\{\left(\mathrm{X}_{\mathrm{PUB}}\right)^{\prime}\left(\beta_{\mathrm{PRI}}-\beta_{\mathrm{PUB}}\right)\right.}_{\text {Efecto interacción }}+\underbrace{\left\{E\left(\mathrm{X}_{\mathrm{PRI}}\right)-E\left(\mathrm{X}_{\mathrm{PUB}}\right)\right\}^{\prime}\left(\beta_{\mathrm{PRI}}-\beta_{\mathrm{PUB}}\right)}}_{\text {Efecto coeficientes }}
$$

Componente no observada

Siendo X el vector de características observables, o dotaciones poseídas, que agrupa a las variables que explican los resultados académicos y $\beta$ el vector de coeficientes, que incluye un término independiente.

1. La primera componente es el denominado "efecto dotaciones", y recoge las diferencias debidas a las distintas características que tienen, en promedio, los individuos de cada grupo. Se calcula como la diferencia en resultados que se produciría en el grupo de alumnos de colegios públicos si sus integrantes

4 Fortin et al. (2011) y Jann (2008) explican exhaustivamente la técnica de descomposición y de su notación. Para un análisis e interpretación detallados, recomendamos recurrir a dichos artículos, así como a los trabajos originales de Oaxaca (1973) y Blinder (1973). La notación que utilizamos y su explicación es una adaptación sintética para el caso abordado. 
tuvieran, en promedio, las mismas características que los integrantes del grupo de alumnos de colegios privados.

2. La segunda componente recoge el "efecto de las diferencias" en los coeficientes, incluido el término independiente. Se calcula como la diferencia en resultados que se produciría en el grupo de alumnos de colegios públicos si sus integrantes obtuvieran los mismos coeficientes que los del grupo de alumnos de colegios privados.

3. Finalmente, la tercera componente es un "término de interacción", que recoge el hecho de que las diferencias tanto en dotaciones como en coeficientes se dan simultáneamente entre los dos grupos.

La función de producción educativa descrita en la ecuación (1) constituye la base sobre la que se aplicará esta descomposición.

\section{Técnicas de estimación utilizadas y puntualizaciones econométricas}

Las técnicas de regresión econométricas más utilizadas para estimar las funciones de producción educativa son la de mínimos cuadrados ordinarios (MCO) y los modelos jerárquicos o multinivel. Estos últimos tienen la ventaja de que permiten tomar en consideración las potenciales correlaciones entre los términos de error que aparecen entre los estudiantes de una misma clase, profesor o escuela. Con todo, el uso de dichos modelos implica la pérdida de grados de libertad y, por consiguiente, tiene efectos sobre la precisión de las estimaciones. Por este motivo, en las estimaciones que se llevan a cabo se utiliza otro método que tiene en cuenta la variabilidad en características observadas y no observadas entre escuelas, al igual que las técnicas multinivel, pero permite obtener estimadores insesgados sin plantear esta pérdida de grados de libertad.

En concreto, se recurre al uso de estimadores MCO con errores estándar corregidos mediante la técnica clúster en función de los centros educativos. Este método contempla la existencia de variables explicativas o características no observables, inherentes a cada uno de los centros y no recogidas por el modelo, que provocarían correlaciones arbitrarias entre los estudiantes pertenecientes a un mismo centro. Si no corrigiéramos esto, tendríamos errores de estimación incorrelacionados entre los diferentes centros pero correlacionados dentro de cada centro. Como consecuencia, las estimaciones de los términos de varianza y covarianza y de los errores estándar asociados a los coeficientes de las estimaciones no tendrían validez. Así, para evitar una mala interpretación del poder explicativo de las variables del modelo, en las tablas de resultados se reportan errores estándar robustos mediante la metodología clúster, usando cada centro 
educativo como variable clúster o grupo de corrección. Además, los errores estándar de los coeficientes obtenidos mediante esta técnica también son robustos ante la presencia de heterocedasticidad.

Otra cuestión metodológica a considerar es que algunas de las variables explicativas utilizadas en las estimaciones son categóricas, lo que afecta a los resultados de la descomposición ов. Como es sabido, este tipo de variables exige asignar un grupo no categorizado, para evitar la multicolinealidad perfecta. La elección arbitraria del grupo de referencia podría generar problemas, ya que si bien el valor general de la descomposición y el componente dotaciones o componente explicado no resultarían afectados sí se producirían cambios en la componente no explicada. Por tanto, se alteraría la parte de esta componente que se atribuye a diferencias en los coeficientes y en los términos independientes, tal y como han apuntado Oaxaca \& Ransom (1999), Horrace \& Oaxaca (2001) o Yun (2005). Para evitar dicho problema, usaremos la técnica propuesta por Jann (2008: p. 10), ${ }^{5}$ que, basada en los trabajos de Gardeazabal \& Ugidos (2004) y Yun (2005), permite que los resultados de la descomposición de OB sean independientes de la elección de la categoría omitida usada como base. Específicamente, planteamos desviaciones en los coeficientes de cada una de las categorías respecto a una media general, de manera que todas sumen cero. Los resultados serían iguales a la media de los resultados que se obtendrían si se aplicara ов utilizando, cada vez, una categoría base diferente.

Así, a partir de la ecuación (1) y teniendo en cuenta estas consideraciones metodológicas, se ha calculado la descomposición ов de los resultados escolares PISA 2012 para los alumnos que asisten a centros públicos y privados costarricenses. Las estimaciones y su interpretación se presentan enseguida.

\section{El análisis empírico: causas de las diferencias en resultados entre colegios costarricenses públicos y privados}

Las tablas 1, 2 y 3 -al final del artículo- contienen los resultados de las descomposiciones ов en las pruebas PISA (2012) de matemáticas, comprensión lectora y ciencias. El análisis permite cuantificar las diferencias de rendimiento entre centros públicos y privados y determinar en qué medida se pueden atribuir a seis elementos: 1) a las diferencias características de los alumnos que acuden a cada tipo de centro (sexo, si cursó preescolar, confianza en sus

5 El autor desarrolla el comando oaxaca para el programa econométrico STATA. Dicho software y comando es el que utilizamos para efectuar las estimaciones, añadiendo la opción categorical, para que los resultados de la descomposición no dependan de la categoría elegida como base.

G. Giménez, G. Castro Aristizábal | ¿Por qué los estudiantes de colegios públicos y privados de Costa Rica obtienen distintos resultados académicos? | Perfiles Latinoamericanos | FLACso México 
capacidades y condición de repetidor); 2) a las diferencias características de los hogares de los que provienen (educación de los padres, riqueza y bienes culturales); 3) a las diferencias en recursos entre centros públicos y privados (profesorado e infraestructuras); 4) a las diferencias en el ambiente de trabajo de los centros públicos y privados (relaciones profesor-alumno, moral del profesorado y disciplina en el centro); 5) al diferente aprovechamiento por parte de los estudiantes de sus características, las de sus hogares y las de los centros donde estudian, y 6) al hecho de que las diferencias tanto en dotaciones como en coeficientes se dan simultáneamente entre los dos grupos.

De acuerdo a la columna 1 de cada tabla, el modelo - estimado, respectivamente, para la muestra de centros públicos y privados_ predice una nota de 413.3 puntos en matemáticas, 481.6 puntos en lectura y 459 puntos en ciencias para los centros privados; y de 376.8, 426.1 y 411.2 puntos para los públicos. Ello se traduce en una diferencia de desempeño de 36.5 puntos en matemáticas, 55.6 en lectura y 47.8 en ciencias.

Las columnas 2, 3 y 4 ofrecen la descomposición detallada del modelo: en los efectos dotaciones o diferencias en características de los alumnos, sus familias y los centros en los que estudian (columna 2); en los efectos coeficientes o aprovechamiento que se hace de estas características (columna 3), y en la interacción entre los dos efectos (columna 4).

Los efectos totales permiten identificar la componente explicada por el modelo (49.5, 48.7 y 44.4 puntos en matemáticas, lectura y ciencias) y la componente no explicada, suma del efecto coeficientes $(-3.2,30.7$ y 18.4 puntos) y el efecto interacción (-9.8, -23.9 y -15 puntos). Al contrastar las componentes explicadas y el efecto neto de las componentes no explicadas, se infiere que el modelo tiene un alto poder explicativo (explica el 74, 88 y $93 \%$ de las diferencias en puntuación en matemáticas, lectura y ciencias).

Las columnas 5, 6 y 7 sintetizan los resultados, para facilitar su análisis, agregando los efectos dotaciones, componentes e interacción de las distintas variables en cuatro grupos, de acuerdo a: 1) características del estudiante, 2) características del hogar, 3) recursos del centro educativo y 4) ambiente de trabajo en el centro. Teniendo en cuenta las diferentes componentes, grupos de variables y pruebas de conocimiento, se pueden extraer las siguientes conclusiones:

\section{A) Componente explicada:}

- La mayor parte de las diferencias en resultados entre centros públicos y privados es explicada por el modelo (efecto dotaciones de la columna 5). La profundización en este efecto permite comprender las causas últimas de las diferencias. 
- Dentro del efecto dotaciones, la principal causa de la divergencia en rendimiento son las características del hogar (que agregan 16.8, 19 y 16.4 puntos a la diferencia final en cada una de las pruebas). Los factores determinantes dentro de esta categoría son, por orden de importancia, el índice de riqueza en el hogar (10.5, 11.9 y 10.2 puntos), los años de educación de la madre (1.7 no significativo, 5.9 y 3.9 puntos) y los años de educación del padre (3.9, 1.5 no significativo y 0.8 no significativo).

— En segundo lugar, el factor más importante son las diferencias en recursos de los centros educativos (que suman 13.2, 13.6 y 14.5 puntos). El elemento principal en esta categoría es el índice de calidad de los recursos educativos del centro (12, 10.9 y 12.1 puntos).

- El tercer factor por orden de importancia engloba las características del estudiante (que suman $12.3,12.7$ y 10.9 puntos). Los factores determinantes dentro de esta categoría son el hecho de no repetir curso $(8.7,8.5$ y 7.8 puntos) y poseer un alto nivel de confianza en sus capacidades matemáticas $(3.8,2.3$ y 2.4$)$.

- El cuarto, y último, es el ambiente de trabajo en los centros (que suma 7.2, 3.4 y 2.5 puntos).

B) Componente no explicada:

- La componente no explicada, compuesta por el efecto coeficientes (columna 6) y el efecto interacción (columna 7) tiene una importancia considerablemente menor en la explicación de las diferencias en rendimiento. Recoge las diferencias en la utilización de los recursos disponibles en los distintos centros públicos y privados, así como el diferente aprovechamiento de las características de los alumnos y los hogares. ${ }^{6}$

- Dentro de la componente no explicada, los efectos en coeficientes de las características del estudiante y del ambiente de trabajo de los centros tienen un mayor peso en la explicación de las diferencias en resultados, atendiendo a su valor y significatividad, pero exclusivamente en las pruebas de matemáticas y ciencias.

- Las características del estudiante restan 16.2 puntos en matemáticas y 21 puntos en ciencias a la diferencia en resultados en dichas pruebas, lo que se interpreta señalando que: aunque los estudiantes de los centros públicos poseen en conjunto peores características o dotaciones factoriales, saben

6 Esto es, cómo podrían aprovechar los alumnos que acuden a centros públicos las características y medios de los alumnos de los centros privados. También estará contabilizando, de manera residual, todos aquellos factores no recogidos por el modelo. 
sacarles mejor partido ${ }^{7}$ (el coeficiente tiene signo negativo). En lectura los efectos no son significativos.

- El ambiente de trabajo de los centros suma 17.6 puntos en matemáticas y 17.8 puntos en ciencias, lo que indica que a los estudiantes de los colegios públicos les influye más negativamente este factor (independientemente del valor de las dotaciones). Nuevamente, en lectura los efectos no resultan significativos.

Por último, hay que indicar que el hecho de que el conjunto de los resultados hallados sea común a las diferentes pruebas académicas los dota de gran robustez.

\section{Conclusiones y recomendaciones en materia de política educativa}

A lo largo del artículo se han puesto de manifiesto las diferencias en resultados académicos entre alumnos costarricenses de centros públicos y privados. La descomposición de Oaxaca-Blinder permite inferir que tales diferencias obedecen a las desiguales dotaciones en los inputs incluidos en la función de producción educativa utilizada en las estimaciones. El principal factor de la divergencia en rendimiento son las características del hogar. Le siguen, por orden de importancia, las diferencias en recursos de los centros educativos, las características del estudiante y el ambiente de trabajo en los centros.

Garantizar la equidad educativa constituye uno de los pilares fundamentales de la política educativa. En este sentido, las medidas diseñadas para reducir las diferencias en la calidad de la formación son prioritarias. Además, este tipo de actuaciones son las más efectivas en el largo plazo para luchar contra la desigualdad de ingresos e incrementar la cohesión social. Del análisis llevado a cabo, se desprende que una política educativa eficaz que garantice la equidad debería ser una política integral y combinada, diseñada desde una triple dimensión:

1) Familia. Es necesario evitar que los niños crezcan en entornos poco estimulantes. Para ello se debe vertebrar una política fiscal progresiva que permita destinar recursos suficientes para cubrir las necesidades de inversión en educación, así como financiar proyectos que eviten el abandono escolar temprano. Este está motivado en muchas ocasiones por la pobreza del hogar. En dicho sentido, hay que destacar el éxito de programas como avanCEMos o proeduca. El primero, a cargo del Instituto Mixto de Ayuda Social de Cos-

7 Aprovechamiento que los estudiantes de los colegios públicos harían de las características de los estudiantes de colegios privados, si las poseyeran. 
ta Rica desde 2006, concede transferencias a estudiantes en situaciones de pobreza extrema, mismas que condiciona a la permanencia o reinserción del estudiante en el sistema de educación secundaria. El segundo, puesto en marcha en 2011 y financiado por el Ministerio de Educación Pública y la Unión Europea, busca disminuir el abandono estudiantil en educación secundaria por medio de distintas acciones de apoyo a estudiantes, profesores, directores y comunidad educativa en general. En otro ámbito, es fundamental asegurar infraestructuras públicas que garanticen que los hogares tienen las necesidades básicas cubiertas en el acceso a infraestructuras sanitarias, eléctricas o de telecomunicaciones. La calidad de este tipo de servicios guarda una fuerte relación con el rendimiento escolar.

2) Escuela. Resulta perentorio reforzar la autoridad de los profesores, capacitarlos, evaluarlos, fomentar su movilidad y estimularlos laboralmente; formar a personal especializado, que pueda identificar a los alumnos con problemas de aprendizaje y ayudarlos brindándoles formación especial; incrementar el número de centros de secundaria en zonas rurales, así como mejorar los recursos de las escuelas en estos entornos; invertir en nuevas tecnologías, a la vez que se cuente con personal formado que sea capaz de sacarles el máximo rendimiento; incrementar la participación de los padres en el entorno escolar, y establecer un marco disciplinario bien definido y común a los distintos centros.

3) Individuo. Las políticas más importantes en términos de equidad pasarían por garantizar el acceso a una educación preescolar de calidad, como forma de fomentar habilidades sociales tempranas y sacar a los niños de ambientes poco estimulantes; incidir en políticas sociales que disminuyan las brechas de género en la infancia; promover el acceso a los niveles de educación secundaria no obligatoria, e implementar políticas de salud pública que garanticen una buena alimentación y atención sanitaria.

Dadas las importantes externalidades positivas que posee la educación, y sus efectos sobre el crecimiento, las inversiones realizadas pueden ser recuperadas con creces en el futuro. 
Tabla 1. Descomposición Oaxaca-Blinder de las diferencias en puntuación entre centros públicos y privados costarricenses en las pruebas de matemáticas PISA (2012)

\begin{tabular}{|c|c|c|c|c|c|}
\hline & & $\begin{array}{c}\text { Efectos } \\
\text { agregados }\end{array}$ & $\begin{array}{c}\text { Descomposición } \\
\text { detallada }\end{array}$ & & \\
\hline & & Diferencia & Dotaciones & Coeficientes & Interacción \\
\hline & & (1) & (2) & (3) & (4) \\
\hline & Predicción centros privados & $413.343^{\star \star \star}$ & & & \\
\hline & & $(10.403)$ & & & \\
\hline & Predicción centros públicos & $376.835^{\star \star \star}$ & & & \\
\hline & & $(5.167)$ & & & \\
\hline & Diferencia & $36.508^{\star \star \star}$ & & & \\
\hline & & $(11.616)$ & & & \\
\hline \multirow{19}{*}{ 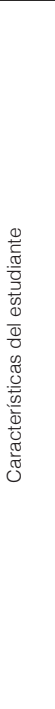 } & Sexo & & & & \\
\hline & Femenino & & 0.074 & $-8.751^{\star \star \star}$ & 0.119 \\
\hline & & & $(0.319)$ & $(2.285)$ & $(0.513)$ \\
\hline & Masculino & & 0.062 & $-6.107^{\star \star}$ & -0.102 \\
\hline & & & $(0.270)$ & $(3.035)$ & $(0.443)$ \\
\hline & Cursó preescolar & & & & \\
\hline & Por un año o menos & & -0.998 & 0.626 & -0.237 \\
\hline & & & $(0.701)$ & $(5.881)$ & $(2.228)$ \\
\hline & Por más de un año & & 0.170 & 6.735 & 5.189 \\
\hline & & & $(1.120)$ & $(5.641)$ & $(4.414)$ \\
\hline & Ante la afirmación: "El estudiante piensa que no es buen & eno en matem & ticas" & & \\
\hline & Está de acuerdo & & -0.011 & -2.528 & 0.676 \\
\hline & & & $(0.252)$ & $(2.241)$ & $(0.637)$ \\
\hline & Está en desacuerdo & & 0.498 & -2.293 & -0.163 \\
\hline & & & $(0.599)$ & $(3.002)$ & $(0.289)$ \\
\hline & Está fuertemente en desacuerdo & & $3.810^{\star \star \star}$ & 1.979 & 1.289 \\
\hline & & & $(1.118)$ & $(1.365)$ & $(0.951)$ \\
\hline & No ha repetido curso & & $8.696^{\star \star \star}$ & -5.880 & -3.051 \\
\hline & & & $(1.905)$ & $(4.345)$ & $(2.332)$ \\
\hline \multirow{8}{*}{ 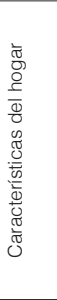 } & Años de estudio de la madre & & 1.698 & 1.927 & 0.898 \\
\hline & & & $(1.626)$ & $(10.306)$ & $(4.805)$ \\
\hline & Años de estudio del padre & & $3.852^{\star \star \star}$ & -9.772 & -3.849 \\
\hline & & & $(1.278)$ & $(10.643)$ & $(4.204)$ \\
\hline & Índice de riqueza en el hogar & & $10.503^{\star \star \star}$ & 1.685 & -1.588 \\
\hline & & & $(2.579)$ & $(6.812)$ & $(6.418)$ \\
\hline & Índice de posesiones culturales en el hogar & & 0.785 & 0.718 & -1.696 \\
\hline & & & $(1.014)$ & $(1.372)$ & $(3.237)$ \\
\hline \multirow{6}{*}{ 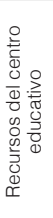 } & Índice de calidad de los recursos educativos del centro & & $11.985^{\star \star}$ & -2.751 & 3.409 \\
\hline & & & $(5.410)$ & $(9.534)$ & $(11.821)$ \\
\hline & Ratio alumnos/profesor & & 1.767 & -2.774 & -1.331 \\
\hline & & & $(3.443)$ & $(5.065)$ & $(3.065)$ \\
\hline & Índice de falta de profesores en el centro & & -0.580 & 0.865 & -3.603 \\
\hline & & & $(0.940)$ & $(1.212)$ & $(3.681)$ \\
\hline
\end{tabular}


Tabla 1. (Continuación)

\begin{tabular}{|c|c|c|c|c|c|}
\hline & & $\begin{array}{c}\text { Efectos } \\
\text { agregados }\end{array}$ & $\begin{array}{c}\text { Descomposición } \\
\text { detallada }\end{array}$ & & \\
\hline & & Diferencia & Dotaciones & Coeficientes & S Interacción \\
\hline & & (1) & (2) & (3) & (4) \\
\hline & Índice de relaciones profesor-alumno & & $1.376^{\star \star \star}$ & 0.788 & -0.413 \\
\hline & & & $(0.483)$ & $(1.217)$ & $(0.647)$ \\
\hline$\stackrel{0}{\geq}$ & El director del centro responde a si los pr & del centro muestr & n una moral alta & & \\
\hline$\frac{0}{0}$ & Está de acuerdo & & $4.117^{\star}$ & $16.709^{*}$ & -6.257 \\
\hline$\stackrel{0}{\circ}$ & & & $(2.352)$ & $(8.977)$ & $(4.576)$ \\
\hline Е్ & Está en desacuerdo & & $0.886^{*}$ & 0.140 & -0.140 \\
\hline$\underset{c}{\bar{\Phi}}$ & & & $(0.535)$ & $(0.327)$ & $(0.327)$ \\
\hline$\frac{\circ}{\pi}$ & Índice de disciplina en el centro & & 0.780 & -0.042 & 1.092 \\
\hline$\stackrel{\frac{0}{\overparen{\pi}}}{=}$ & & & $(0.681)$ & $(0.143)$ & $(1.524)$ \\
\hline$\frac{1}{0}$ & Constante & & & 5.522 & \\
\hline 㫕 & & & & $(31.114)$ & \\
\hline है & Total & & $49.471^{\star \star \star}$ & -3.205 & -9.757 \\
\hline & & & $(8.094)$ & (18.484) & (17.993) \\
\hline & Número de alumnos & 1,849 & & & \\
\hline & & & Descomposición deta & allada & \\
\hline & & Dotaciones & Coeficientes & & nteracción \\
\hline & & (5) & (6) & & (7) \\
\hline Tot & & $49.471^{\star \star \star}$ & -3.205 & & -9.757 \\
\hline & & (8.094) & $(18.484)$ & & (17.993) \\
\hline & terísticas del estudiante & $12.302^{\star \star \star}$ & $-16.219^{*}$ & & 3.719 \\
\hline & & $(2.641)$ & $(8.994)$ & & $(4.309)$ \\
\hline & terísticas del hogar & $16.838^{\star \star \star}$ & -5.442 & & -6.234 \\
\hline & & (2.996) & (17.475) & & (8.066) \\
\hline & sos del centro educativo & $13.171^{\star *}$ & -4.661 & & -1.525 \\
\hline & & (5.709) & $(11.050)$ & & $(11.512)$ \\
\hline & ente de trabajo en el centro educativo & $7.159^{\text {*** }}$ & $17.595^{*}$ & & -5.717 \\
\hline & & $(2.377)$ & $(9.059)$ & & $(5.266)$ \\
\hline & ante & & 5.522 & & \\
\hline & & & $(31.114)$ & & \\
\hline & ro de alumnos & 1,849 & & & \\
\hline
\end{tabular}

Significatividad: ${ }^{* *} \mathrm{p}<0.01,{ }^{* *} \mathrm{p}<0.05,{ }^{*} \mathrm{p}<0.1$. Errores estándar entre paréntesis. Estimaciones $\mathrm{MCO}$ con errores estándar robustos corregidos mediante la técnica de Huber-White, utilizando clústeres por centros educativos. Observaciones ponderadas de acuerdo a sus pesos muestrales. Variable dependiente estimada en función de la media de valores plausibles.

Fuente: Elaboración propia. 
Tabla 2. Descomposición Oaxaca-Blinder de las diferencias en puntuación entre centros públicos y privados costarricenses en las pruebas de comprensión lectora PISA (2012)

\begin{tabular}{|c|c|c|c|c|c|}
\hline & & Efectos agregados & $\begin{array}{c}\text { Descomposición } \\
\text { detallada }\end{array}$ & & \\
\hline & & Diferencia & Dotaciones & Coeficientes & Interacción \\
\hline & & (1) & (2) & (3) & (4) \\
\hline & Predicción centros privados & $481.612^{\star \star \star}$ & & & \\
\hline & & $(14.228)$ & & & \\
\hline & Predicción centros públicos & $426.060^{\star \star \star}$ & & & \\
\hline & & $(7.061)$ & & & \\
\hline & Diferencia & $55.552^{* \star *}$ & & & \\
\hline & & $(15.884)$ & & & \\
\hline \multirow{19}{*}{ 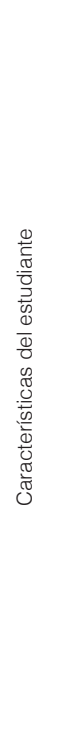 } & \multicolumn{5}{|l|}{ Sexo } \\
\hline & \multirow{2}{*}{\multicolumn{2}{|c|}{ Femenino }} & 0.034 & -4.710 & 0.064 \\
\hline & & & $(0.147)$ & $(2.987)$ & $(0.279)$ \\
\hline & \multirow{2}{*}{\multicolumn{2}{|c|}{ Masculino }} & -0.276 & 1.451 & 0.024 \\
\hline & & & $(1.194)$ & $(4.291)$ & $(0.127)$ \\
\hline & \multicolumn{5}{|l|}{ Cursó preescolar } \\
\hline & \multirow{2}{*}{\multicolumn{2}{|c|}{ Por un año o menos }} & $-1.993^{*}$ & -5.021 & 1.901 \\
\hline & & & $(1.024)$ & $(6.292)$ & $(2.425)$ \\
\hline & \multirow{2}{*}{\multicolumn{2}{|c|}{ Por más de un año }} & $3.685^{\star *}$ & -2.488 & -1.917 \\
\hline & & & $(1.780)$ & $(5.194)$ & $(4.012)$ \\
\hline & \multicolumn{5}{|c|}{ Ante la afirmación: "El estudiante piensa que no es bueno en matemáticas" } \\
\hline & \multirow{2}{*}{\multicolumn{2}{|c|}{ Está de acuerdo }} & 0.140 & 0.718 & -0.192 \\
\hline & & & $(0.342)$ & $(3.054)$ & $(0.819)$ \\
\hline & \multirow{2}{*}{\multicolumn{2}{|c|}{ Está en desacuerdo }} & 0.279 & 0.843 & 0.060 \\
\hline & & & $(0.350)$ & $(4.937)$ & $(0.358)$ \\
\hline & \multirow{2}{*}{\multicolumn{2}{|c|}{ Está fuertemente en desacuerdo }} & $2.296^{* *}$ & 2.782 & 1.812 \\
\hline & & & $(0.823)$ & $(2.624)$ & $(1.774)$ \\
\hline & \multirow{2}{*}{\multicolumn{2}{|c|}{ No ha repetido curso }} & $8.532^{* *}$ & -2.572 & -1.335 \\
\hline & & & $(1.924)$ & $(4.903)$ & $(2.558)$ \\
\hline \multirow{8}{*}{ 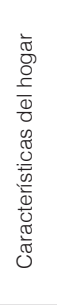 } & \multirow{2}{*}{\multicolumn{2}{|c|}{ Años de estudio de la madre }} & $5.904^{* \star *}$ & -5.161 & -2.406 \\
\hline & & & $(1.815)$ & $(9.513)$ & $(4.437)$ \\
\hline & \multirow{2}{*}{\multicolumn{2}{|c|}{ Años de estudio del padre }} & 1.514 & 2.101 & 0.827 \\
\hline & & & $(1.613)$ & $(13.222)$ & $(5.208)$ \\
\hline & \multirow{2}{*}{\multicolumn{2}{|c|}{ Índice de riqueza en el hogar }} & $11.947^{\star \star \star}$ & 5.750 & -5.416 \\
\hline & & & $(3.183)$ & $(6.507)$ & $(6.146)$ \\
\hline & \multirow{2}{*}{\multicolumn{2}{|c|}{ Índice de posesiones culturales en el hogar }} & -0.375 & -0.036 & 0.085 \\
\hline & & & $(1.253)$ & $(1.081)$ & $(2.554)$ \\
\hline \multirow{6}{*}{ 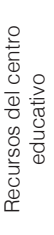 } & \multirow{2}{*}{\multicolumn{2}{|c|}{ Índice de calidad de los recursos educativos del centro }} & $10.904^{* *}$ & 5.904 & -7.317 \\
\hline & & & $(5.154)$ & $(9.601)$ & $(11.927)$ \\
\hline & \multirow{2}{*}{\multicolumn{2}{|c|}{ Ratio alumnos/profesor }} & 3.675 & $-8.366^{\star}$ & -4.014 \\
\hline & & & $(5.665)$ & $(5.023)$ & $(6.126)$ \\
\hline & \multirow{2}{*}{\multicolumn{2}{|c|}{ Índice de falta de profesores en el centro }} & -0.973 & 0.573 & -2.389 \\
\hline & & & (1.154) & (0.918) & (3.063) \\
\hline
\end{tabular}


Tabla 2. (Continuación)

\begin{tabular}{|c|c|c|c|c|c|}
\hline & & Efectos agregados & $\begin{array}{c}\text { Descomposicic } \\
\text { detallada }\end{array}$ & & \\
\hline & & Diferencia & Dotaciones & Coeficientes & Interacción \\
\hline & & (1) & (2) & (3) & (4) \\
\hline & $\begin{array}{l}\text { Índice de relaciones profesor- } \\
\text { alumno }\end{array}$ & & $0.930^{* *}$ & -0.423 & 0.222 \\
\hline స్이 & & & $(0.449)$ & $(1.354)$ & $(0.712)$ \\
\hline 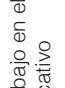 & $\begin{array}{l}\text { El director del centro responde a si } \\
\text { los profesores del centro muestran } \\
\text { una moral alta }\end{array}$ & & & & \\
\hline$\stackrel{0}{5}$ & Está de acuerdo & & 1.195 & $13.970^{*}$ & -5.231 \\
\hline 0 & & & $(1.503)$ & $(7.943)$ & (3.948) \\
\hline$\frac{\bar{\sigma}}{0}$ & Está en desacuerdo & & 0.068 & -0.311 & 0.311 \\
\hline है & & & $(0.278)$ & $(0.346)$ & $(0.346)$ \\
\hline & Índice de disciplina en el centro & & 1.246 & -0.040 & 1.041 \\
\hline & & & $(0.839)$ & $(0.138)$ & $(1.561)$ \\
\hline & Constante & & & 25.727 & \\
\hline & & & & $(28.706)$ & \\
\hline & Total & & $48.731^{\star \star \star}$ & $30.690^{*}$ & -23.869 \\
\hline & & & (9.352) & $(18.391)$ & $(16.967)$ \\
\hline & Número de alumnos & 1,849 & & & \\
\hline & & & Descomposici & ón detallada & \\
\hline & & Dotacio & & Coeficientes & Interacción \\
\hline & & (5) & & (6) & (7) \\
\hline Total & & 48.7 & & $30.690^{*}$ & -23.869 \\
\hline & & $(9.3$ & & $(18.391)$ & $(16.967)$ \\
\hline Caract & terísticas del estudiante & 12.6 & & -8.998 & 0.418 \\
\hline & & $(2.4$ & & $(7.513)$ & $(4.093)$ \\
\hline Caract & terísticas del hogar & 18.9 & & 2.654 & -6.910 \\
\hline & & (3.3 & & $(16.656)$ & $(7.160)$ \\
\hline Recurs & sos del centro educativo & 13.6 & & -1.889 & -13.720 \\
\hline & & $(7.6$ & & $(10.095)$ & $(13.771)$ \\
\hline Ambie & nte de trabajo en el centro educativo & 3.4 & & 13.196 & -3.658 \\
\hline & & $(1.7$ & & $(8.237)$ & (4.605) \\
\hline Consta & ante & & & 25.727 & \\
\hline & & & & $(28.706)$ & \\
\hline Númer & o de alumnos & 1,849 & & & \\
\hline
\end{tabular}

Significatividad: ${ }^{* *} p<0.01,{ }^{* *} p<0.05,{ }^{*} p<0.1$. Errores estándar entre paréntesis. Estimaciones MCO con errores estándar robustos corregidos mediante la técnica de Huber-White, utilizando clústeres por centros educativos. Observaciones ponderadas de acuerdo a sus pesos muestrales. Variable dependiente estimada en función de la media de valores plausibles.

Fuente: Elaboración propia. 
Tabla 3. Descomposición Oaxaca-Blinder de las diferencias en puntuación entre centros públicos y privados costarricenses en las pruebas de ciencias PISA (2012)

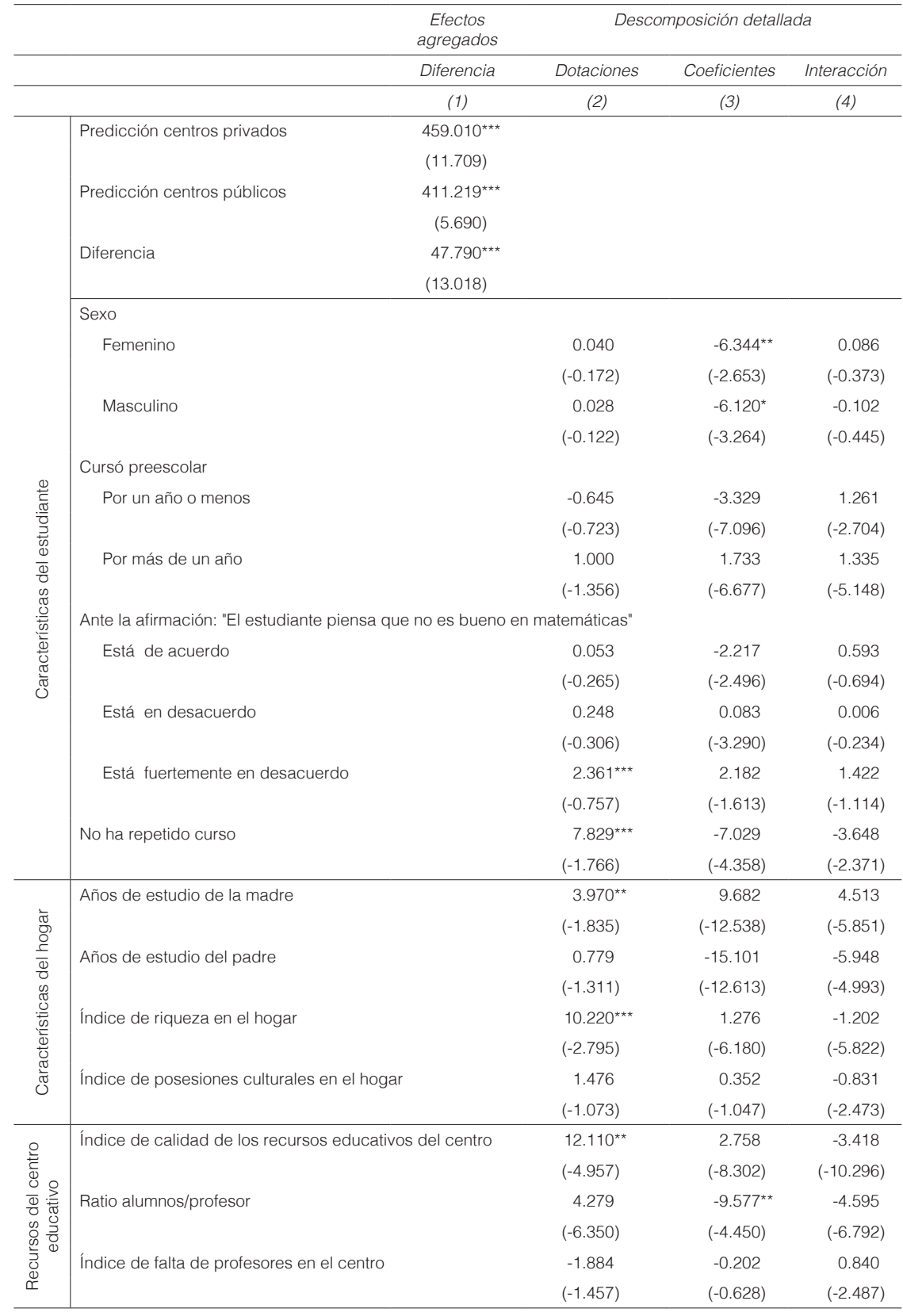


Tabla 3. (Continuación)

\begin{tabular}{|c|c|c|c|c|c|}
\hline & & \multirow{2}{*}{$\begin{array}{c}\begin{array}{c}\text { Efectos } \\
\text { agregados }\end{array} \\
\text { Diferencia }\end{array}$} & \multicolumn{3}{|c|}{ Descomposición detallada } \\
\hline & & & Dotaciones & Coeficientes & Interacción \\
\hline & & (1) & (2) & (3) & (4) \\
\hline \multirow{14}{*}{ 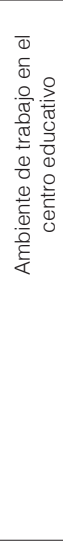 } & Índice de relaciones profesor-alumno & & $1.062^{\star \star}$ & 0.345 & -0.181 \\
\hline & & & $(-0.441)$ & $(-1.201)$ & $(-0.631)$ \\
\hline & \multicolumn{5}{|c|}{ El director del centro responde a si los profesores del centro muestran una moral alta } \\
\hline & \multirow[t]{2}{*}{ Está de acuerdo } & & 1.052 & $17.243^{\star \star}$ & -6.457 \\
\hline & & & $(-1.340)$ & $(-7.536)$ & $(-4.270)$ \\
\hline & \multicolumn{2}{|l|}{ Está en desacuerdo } & 0.735 & 0.247 & -0.247 \\
\hline & & & $(-0.497)$ & $(-0.379)$ & $(-0.379)$ \\
\hline & \multicolumn{2}{|l|}{ Índice de disciplina en el centro } & -0.331 & -0.060 & 1.581 \\
\hline & & & $(-0.598)$ & $(-0.196)$ & $(-1.406)$ \\
\hline & \multirow{2}{*}{\multicolumn{2}{|c|}{ Constante }} & & 32.480 & \\
\hline & & & & $(31.994)$ & \\
\hline & \multirow[t]{2}{*}{ Total } & & $44.382^{\star \star \star}$ & 18.402 & -14.994 \\
\hline & & & $(-9.985)$ & $(-18.689)$ & $(-17.088)$ \\
\hline & \multirow[t]{4}{*}{ Número de alumnos } & \multicolumn{4}{|l|}{1,849} \\
\hline & & \multicolumn{4}{|c|}{ Descomposición detallada } \\
\hline & & Dotaciones & \multicolumn{2}{|c|}{ Coeficientes } & Interacción \\
\hline & & (5) & \multicolumn{2}{|c|}{ (6) } & $(7)$ \\
\hline \multirow{2}{*}{\multicolumn{2}{|c|}{ Total }} & $44.382^{\star \star \star}$ & \multicolumn{2}{|c|}{18.402} & -14.994 \\
\hline & & $(9.985)$ & \multicolumn{2}{|c|}{$(18.689)$} & $(17.088)$ \\
\hline \multirow{2}{*}{\multicolumn{2}{|c|}{ Características del estudiante }} & $10.914^{\star \star \star}$ & \multicolumn{2}{|c|}{$-21.042^{* *}$} & 0.952 \\
\hline & & $(2.268)$ & \multicolumn{2}{|c|}{$(8.901)$} & $(3.821)$ \\
\hline \multirow{2}{*}{\multicolumn{2}{|c|}{ Características del hogar }} & $16.446^{* * *}$ & \multicolumn{2}{|c|}{-3.791} & -3.468 \\
\hline & & (2.910) & \multicolumn{2}{|c|}{$(18.004)$} & $(7.306)$ \\
\hline \multirow{2}{*}{\multicolumn{2}{|c|}{ Recursos del centro educativo }} & $14.505^{*}$ & \multicolumn{2}{|c|}{-7.020} & -7.174 \\
\hline & & $(7.842)$ & & 93) & $(12.777)$ \\
\hline Ambie & nte de trabajo en el centro educativo & 2.517 & & $75^{\star \star}$ & -5.304 \\
\hline & & (1.552) & & & $(4.770)$ \\
\hline Conste & ante & & & & \\
\hline & & & & & \\
\hline Númer & o de alumnos & 1,849 & & & \\
\hline
\end{tabular}

Significatividad: ${ }^{\star * *} \mathrm{p}<0.01,{ }^{* *} \mathrm{p}<0.05,{ }^{*} \mathrm{p}<0.1$. Errores estándar entre paréntesis. Estimaciones $\mathrm{MCO}$ con errores estándar robustos corregidos mediante la técnica de Huber-White, utilizando clústeres por centros educativos. Observaciones ponderadas de acuerdo a sus pesos muestrales. Variable dependiente estimada en función de la media de valores plausibles.

Fuente: Elaboración propia. 


\section{Referencias}

Altonji, J. G., Todd, E. E. \& Taber, C. R. (2005). Selection on Observed and Unobserved Variables: Assessing the Effectiveness of Catholic Schools. Journal of Political Economy, 113(1), 151-184.

Bellei, C., Valenzuela, J. P., Sevilla, A. \& Osses, A. (2009). Que explica las diferencias de resultados PISA Lectura entre Chile y algunos países de la OCDE y América Latina. En Que nos dice PISA sobre la educación de los jóvenes en Chile? Nuevos análisis y perspectivas sobre los resultados en PISA 2006. Santiago, Chile: Ministerio de Educación, Unidad de Currículum y Evaluación.

Benton, T. (2014). A Re-Evaluation of the Link between Autonomy, Accountability and Achievement in PISA 2009. Research Division. Assessment Research and Development, Cambridge Assessment. Cambridge, Inglaterra.

Bishop, J. H. \& Woessmann, L. (2004). Institutional Effects in a Simple Model of Educational Production. Education Economics, 12(1), 17-38.

Blinder, A. S. (1973). Wage Discrimination: Reduced Form and Structural Estimates. Journal of Human Resources, 8(4), 436-455.

Burtless, G. T. (ed.). (1996). Does Money Matter? The Effect of School Resources on Student Achievement and Adult Success. Washington, D. C.: Brookings Institution Press.

Calero, J. \& Escardíbul, J. O. (2007). Evaluación de servicios educativos. El rendimiento en los centros públicos y privados medido en PISA-2003. Hacienda Pública Española, 183(4), 33-66.

Cohen-Zada, D. \& William, S. (2007). Religion, Religiosity and Private School-Choice: Implications for Estimating the Effectiveness of Private Schools. Journal of Urban Economics, 64(1), 85-100.

Coleman, J. S. \& Hoffer, T. (1987). Public and Private High Schools: The Impact of Communities. Nueva York: Basic Books.

Coleman, J., Hoffer, T. \& Kilgore, S. (1982). Cognitive Outcomes in Public and Private Schools. Sociology of Education, 55(2), 65-76.

Dearden, L., Chris, R. \& Luke, S. (2010). What Determines Private School Choice? A Comparison between the UK and Australia. Technical report, IFS working papers.

Dronkers, J. \& Robert, P. (2005). School Choice in the Light of the Effectiveness Differences of Various Types of Public and Private School in 19 OECD Countries. MPRA Paper 21888, University Library of Munich, Germany. 
Fernández, A. \& Del Valle, R. (2013). Desigualdad educativa en Costa Rica: la brecha entre estudiantes de colegios públicos y privados. Análisis con los resultados de la evaluación internacional PISA. Revista CEPAL, (11), 37-57.

Figlio, D. N. \& Stone, J. A. (2001). Can Public Policy Affect Private School Cream Skimming?. Journal of Urban Economics, (49), 240-266.

Formichella, M. M. (2011). Do Private Schools in Argentina Perform better because They Are Private?. Cepal Review, (105), 141-155.

Fortin, N., Lemieux, T. \& Firpo, S. (2011). Decomposition Methods in Economics. En Ashenfelter, O. \& Card, D. (Eds.). Handbook of Labor Economics (pp. 1-102), 1 edición, vol. 4, núm. 4. Amsterdam, Holland: Elsevier.

Fuchs, T. \& Woessmann, L. (2007). What Accounts for International Differences in Student Performance? A Re-Examination Using PISA Data. Empirical Economics, (32), 433-464.

Gardeazabal, J. \& Ugidos, A. (2004). More on Identification in Detailed Wage Decompositions. Review of Economics and Statistics, (86), 1034-1036.

Gamoran, A. (1996). Student Achievement in Public Magnet, Public Comprehensive, and Private City High Schools. Educational Evaluation and Policy Analisys, 18 (1), 1-18.

Green, F., Machin, S., Murphy, R. \& Zhu, Y. (2012). The Changing Economic Advantage from Private Schools. Economica, (79), 658-679.

Hanushek, E. A. (2005). The Economics of School Quality. German Economic Review, Verein für Socialpolitik, 6(3), 269-286.

Hanushek, E. A., Link, S. \& Woessmann, L. (2013). Does School Autonomy Make Sense Everywhere? Panel Estimates from PISA. Journal of Development Economics, (104), 212-232.

Hanushek, E. A. \& Welch, F. (Eds.). (2006). Handbook of the Economics of Education. Amsterdam, North-Holland.

Harris, D. N. (2010). Education Production Functions: Concepts. En McGraw, B., Peterson, P. \& Baker, E. (Eds.). International Encyclopedia of Education, (pp. 127-131). Elsevier, Amsterdam.

Horrace, W. C. \& Oaxaca, R. L. (2001). Inter-Industry Wage Differentials and the Gender Wage Gap: An Identification Problem. Industrial and Labor Relations Review, (54), 611-618.

Jann, B. (2008). The Blinder-Oaxaca Decomposition for Linear Regression Models. The Stata Journal, 8(4), 453-479.

G. Giménez, G. Castro Aristizábal | ¿Por qué los estudiantes de colegios públicos y privados de Costa Rica obtienen distintos resultados académicos? | Perfiles Latinoamericanos | FLACso México 
Jimenez, E. \& Cox, D. (1991). The Relative Effectiveness of Private and Public Schools: Evidence from Two Developing Countries. Journal of Developmental Economics, (34), 99-121.

Jimenez, E., Lockheed, M. E. \& Paqueo, V. (1991). The Relative Efficiency of Private and Public Schools in Developing Countries. World Bank Research Observer, 6(2), 205-218.

Kirjavainen, T. \& Loikkanen, H. (1998). Efficiency Differences of Finnish Senior Secondary Schools: An Application of DEA and Tobit Analysis. Economics of Education Review, (17), 377-394.

Krueger, A. B. (1999). Experimental Estimates Of Education Production Functions. The Quarterly Journal of Economics, 114(2), 497-532.

Lassibille, G. \& Tan, J. P. (2001). Are Private Schools More Efficient Than Public Schools? Evidence from Tanzania. Education Economics, 9(2), 145-172.

Levin, J. D. (2002). Essays in the Economics of Education. Tesis doctoral, University of Amsterdam.

Lockheed, M. E. \& Bruns, B. (1990). School Effects on Achievement in Secondary Mathematics and Portuguese in Brazil. Washington, D. C.: Banco Mundial.

Megabase de datos georreferenciados de centros educativos de Costa Rica (2000-2012). San José, Costa Rica: Estado de la Educación.

Montero, E., Rojas, S., Zamora, E. \& Rodino, A. M. (2012). Costa Rica en las pruebas PISA 2009 de competencia lectora y alfabetización matemática. Informe preparado para el Cuarto Informe Estado de la Educación. San José, Costa Rica: Programa Estado de la Educación.

Neal, D. (1997). The Effects of Catholic Secondary Schooling on Educational Achievement. Journal of Labor Economics, (15), 98-123.

Newhouse, D. \& Beegle, K. (2006). The Effect School Type on Academic Achievement. The Journal of Human Resources, 41(3), 529-557.

Oaxaca, R. L. (1973). Male-Female Wage Differentials in Urban Labor Markets. International Economic Review, 14(3), 693-709.

Oaxaca, R. L. \& Ransom, M. R. (1999). Identification in Detailed Wage Decompositions. The Review of Economics and Statistics, 81(1), 154-157.

oCDE. (2011). Private Schools: Who Benefits?. PISA In Focus, París.

OCDE. (2014). When is Competition between Schools Benefical?. PISA In Focus, París. 
Oliveira de, P. R., Belluzzo, W. \& Pazello, E. T. (2013). The Public-Private Test Score Gap in Brazil. Economics of Education Review, 35(C), 120-133.

Oreiro, C. \& Valenzuela, J. P. (2012). Factores que determinan el desempeño educativo en el Uruguay, 2003-2006. Cepal Review, (107), 63-86.

Ramos, R., Duque, J. C.\& Nieto, S. (2012). Un análisis de las diferencias rurales y urbanas en el rendimiento educativo de los estudiantes colombianos a partir de los microdatos de PISA. En Pacheco Vieira, E. (Ed.). Investigaciones de Economía de la Educación, (7), 775-796.

Sánchez, A. (2011). Etnia y Desempeño Académico en Colombia. Documentos de Trabajo sobre Economía Regional, (156). Cartagena, Colombia: Banco de la República, Centro de Estudios Económicos Regionales (CEER).

Sander, W. \& Cohen-Zada, D. (2010). The Economics of Catholic Schools. En McGraw, B., Peterson, P. \& Baker, E. (Eds.). International Encyclopedia of Education (pp. 317-322). Amsterdam: Elsevier.

Schultz, M. M. \& McDonald, D. (2013). United States Catholic Elementary and Secondary Schools 2012-2013. Washington, DC.: National Catholic Educational Association.

Steinberg, M. P. (2014). Does Greater Autonomy Improve School Performance? Evidence from a Regression Discontinuity Analysis in Chicago. Education Finance and Policy, 9(1), 1-35.

Valenzuela, J. P., Bellei, C., Sevilla, A. \& Osses, A. (2009). Qué explica las diferencias de resultados PISA Matemática entre Chile y algunos países de la OCDE y América Latina. En ¿Qué nos dice PISA sobre la educación de los jóvenes en Chile? Nuevos análisis y perspectivas sobre los resultados en PISA 2006. Santiago, Chile: Ministerio de Educación, Unidad de Currículum y Evaluación.

Vella, F. (1999). Do Catholic Schools Make a Difference? Evidence from Australia. Journal of Human Resources, 34(1), 208-224.

West, M. R. \& Woessmann, L. (2010). "Every Catholic Child in a Catholic School”: Historical Resistance to State Schooling, Contemporary Private Competition and Student Achievement across Countries. Economic Journal, 120(546), F229-F255.

Woessmann, L., Luedemann, E., Schutz, G. \& West, M. R. (2009). School Accountability, Autonomy, and Choice Around the World. Cheltenham, UK: Edward Elgar.

Yun, M. S. (2005). A Simple Solution to the Identification Problem in Detailed Wage Decompositions. Economic Inquiry, (43), 766-772.

G. Giménez, G. Castro Aristizábal | ¿Por qué los estudiantes de colegios públicos y privados de Costa Rica obtienen distintos resultados académicos? | Perfiles Latinoamericanos | FLACso México 


\section{Apéndice. Descripción y principales estadísticos de las variables empleadas en el análisis empírico}

Fuente de las variables: Program for International Student Assessment (PISA) base 2012.

\begin{tabular}{|c|c|c|c|c|c|}
\hline Variable & Observ. & Media & Desv. Est. & Mín. & Máx. \\
\hline $\begin{array}{l}\text { Ante la afirmación: "El estudiante piensa que no es bueno en } \\
\text { matemáticas", el estudiante }\end{array}$ & 2877 & & & & \\
\hline Está muy de acuerdo & \multicolumn{5}{|c|}{ (Categoría base) } \\
\hline Está de acuerdo & & 0.26 & 0.44 & 0 & 1.00 \\
\hline Está en desacuerdo & & 0.37 & 0.48 & 0 & 1.00 \\
\hline Está fuertemente en desacuerdo & & 0.19 & 0.39 & 0 & 1.00 \\
\hline $\begin{array}{l}\text { Años de estudio de la madre. Calculados a partir del nivel } \\
\text { educativo alcanzado por la progenitora. }\end{array}$ & 4521 & 10.13 & 4.89 & 0 & 15.50 \\
\hline $\begin{array}{l}\text { Años de estudio del padre. Calculados a partir del nivel edu- } \\
\text { cativo alcanzado por el progenitor. }\end{array}$ & 4214 & 10.25 & 4.91 & 0 & 15.50 \\
\hline Carácter del centro & 4602 & & & & \\
\hline Público & & \multicolumn{2}{|c|}{ (Categoría base) } & & \\
\hline Privado & & 0.15 & 0.36 & 0 & 1.00 \\
\hline Cursó preescolar & 4507 & & & & \\
\hline No & & (base) & & & \\
\hline Por un año o menos & & 0.40 & 0.49 & 0 & 1.00 \\
\hline Por más de un año & & 0.45 & 0.50 & 0 & 1.00 \\
\hline $\begin{array}{l}\text { El director del centro responde a si los profesores del centro } \\
\text { muestran una moral alta }\end{array}$ & 4527 & & & & \\
\hline Está muy de acuerdo & & \multicolumn{2}{|c|}{ (Categoría base) } & & \\
\hline Está de acuerdo & & 0.53 & 0.50 & 0 & 1.00 \\
\hline Está en desacuerdo & & 0.04 & 0.19 & 0 & 1.00 \\
\hline Está fuertemente en desacuerdo & & 0.00 & 0.07 & 0 & 1.00 \\
\hline $\begin{array}{l}\text { Índice de calidad de los recursos educativos del centro. } \\
\text { Se construye con base en la percepción del director del centro } \\
\text { sobre siete elementos que podrían obstaculizar el proceso } \\
\text { educativo: Internet, computadoras, software, recursos biblio- } \\
\text { gráficos y audiovisuales, laboratorios de ciencias y materiales } \\
\text { educativos. } \\
\text { Un mayor valor indica una mayor calidad de los recursos. } \\
\text { Para una explicación exhaustiva del índice, véase OECD } \\
\text { (2012), PISA 2009 Technical Report, PISA, OECD Publishing. } \\
\text { http://dx.doi.org/10.1787/9789264167872-en }\end{array}$ & 4589 & -1.08 & 1.24 & -3.59 & 1.98 \\
\hline $\begin{array}{l}\text { Índice de disciplina en el centro. } \\
\text { Brinda información acerca de la disciplina dentro de las aulas. } \\
\text { Un mayor valor indica mayor disciplina. } \\
\text { Para una explicación exhaustiva del índice, véase OECD } \\
\text { (2012), PISA 2009 Technical Report, PISA, OECD Publishing. } \\
\text { http://dx.doi.org/10.1787/9789264167872-en }\end{array}$ & 2890 & 0.04 & 0.88 & -2.48 & 1.85 \\
\hline $\begin{array}{l}\text { Índice de falta de profesores en el centro. } \\
\text { Se construye en base a la percepción del director del centro } \\
\text { sobre una serie de elementos que podrían obstaculizar el } \\
\text { proceso de enseñanza: falta de profesores cualificados en } \\
\text { matemáticas, lengua, ciencias y otras disciplinas académicas. } \\
\text { Un mayor valor indica mayores carencias en el centro. } \\
\text { Para una explicación exhaustiva del índice, véase OECD } \\
\text { (2012), PISA 2009 Technical Report, PISA, OECD Publishing. } \\
\text { http://dx.doi.org/10.1787/9789264167872-en }\end{array}$ & 4566 & -0.01 & 0.84 & -1.09 & 2.45 \\
\hline
\end{tabular}

222 • G. Giménez, G. Castro Aristizábal | ¿Por qué los estudiantes de colegios públicos y privados de Costa Rica obtienen distintos resultados académicos? | Perfiles Latinoamericanos | Flacso México 


\begin{tabular}{|c|c|c|c|c|c|}
\hline Variable & Observ. & Media & Desv. Est. & Mín. & Máx. \\
\hline $\begin{array}{l}\text { Índice de posesiones culturales en el hogar. } \\
\text { Indica si el hogar está en posesión de una serie de bienes } \\
\text { culturales: libros de literatura clásica y poesía y obras de arte. } \\
\text { Un mayor valor indica más posesiones culturales. } \\
\text { Para una explicación exhaustiva del índice, véase OECD } \\
\text { (2012), PISA 2009 Technical Report, PISA, OECD Publishing. } \\
\text { http://dx.doi.org/10.1787/9789264167872-en }\end{array}$ & 4441 & -0.22 & 0.96 & -1.51 & 1.27 \\
\hline $\begin{array}{l}\text { Índice de relaciones profesor-alumno } \\
\text { Este índice provee información acerca del interés del profesor } \\
\text { en el alumno. Un mayor valor se traduce como mejores relacio- } \\
\text { nes profesor-alumno. } \\
\text { Para una explicación exhaustiva del índice, véase OECD } \\
\text { (2012), PISA 2009 Technical Report, PISA, OECD Publishing. } \\
\text { http://dx.doi.org/10.1787/9789264167872-en }\end{array}$ & 2483 & 0.50 & 1.07 & -3.11 & 2.16 \\
\hline $\begin{array}{l}\text { Índice de riqueza en el hogar. } \\
\text { Indica si el hogar está en posesión de una serie de bienes } \\
\text { materiales, como televisores, vehículos o computadoras, entre } \\
\text { otros. } \\
\text { Un mayor valor indica mayor riqueza. } \\
\text { Para una explicación exhaustiva del índice, véase OECD } \\
\text { (2012), PISA 2009 Technical Report, PISA, OECD Publishing. } \\
\text { http://dx.doi.org/10.1787/9789264167872-en }\end{array}$ & 4500 & -1.34 & 1.14 & -5.04 & 2.88 \\
\hline Variable & Observ. & Media & Desv. Est. & Mín. & Máx. \\
\hline No ha repetido curso & 4602 & 0.47 & 0.50 & 0 & 1.00 \\
\hline Ratio alumnos-profesor & 3483 & 20.40 & 24.29 & 1.55 & 228.60 \\
\hline Resultados Matemáticas (como media de valores plausibles) & 4602 & 407.00 & 64.72 & 195.21 & 694.74 \\
\hline Resultados Lectura (como media de valores plausibles) & 4602 & 440.55 & 69.21 & 196.38 & 660.55 \\
\hline Resultados Ciencias (como media de valores plausibles) & 4602 & 429.35 & 65.04 & 189.24 & 674.60 \\
\hline Sexo & 4602 & & & & \\
\hline Femenino & & \multicolumn{2}{|c|}{ (Categoría base) } & & \\
\hline Masculino & & 0.47 & 0.50 & 0 & 1.00 \\
\hline
\end{tabular}

Recibido el 1 de marzo de 2015. Aceptado el 13 de octubre de 2015. 